\title{
Functional lid surgery in Down's syndrome
}

\author{
R J MORRIS AND J R O COLLIN \\ From Moorfields Eye Hospital, City Road, London EC1
}

SUMMARY Down's syndrome is associated with characteristic eyelid abnormalities. Ten patients presented with lid abnormalities leading to corneal problems. Five patients had ectropion, four of whom required skin grafting to correct the abnormality. One patient had entropion and four had chronic blepharoconjunctivitis leading to trichiasis, which was treated with double freeze thaw cryotherapy.

Down's syndrome is associated with a wide variety of anatomical and biochemical abnormalities including congenital mental retardation, congenital heart defects, immune-system deficiencies, and ocular abnormalities-most commonly cataracts, keratoconus, Brushfield spots, and strabismus. The incidence of the disease is approximately 1 in 700 live births and becomes greater with increasing parental age. ${ }^{1}$ The characteristic facial features associated with Down's syndrome include macroglossia, hypotonia of the lower lip, receding chin, hypoplasia of the nasal bridge, epicanthic folds, oblique lid axis, and dysplastic ears. In 1977 Hohler described a case of a girl with Down's syndrome in whom he undertook facial plastic surgery purely for cosmesis, ${ }^{2}$ and since then there have been other reports describing the results of cosmetic facial plastic surgery for Down's syndrome..$^{3-6}$ In some cases however, the characteristic lid abnormalities may require correction for functional reasons. In particular, ectropion due to skin shortage may lead to corneal exposure, and trichiasis resulting from chronic blepharitis may similarly lead to corneal problems.

We report on 10 patients who presented with lid abnormalities leading to corneal problems, nine of whom underwent lid surgery.

\section{Patients and methods}

Fifty four patients with Down's syndrome who had attended the hospital were studied; of these 10 had eyelid problems directly related to their disease, and the rest had other congenital ocular abnormalities. There were five male and five female patients. The age range at presentation was 7 months to 55 years.

Correspondence to J R O Collin, Moorfields Eye Hospital, City Road, London EC1V 2PD.
ECTROPION GROUP (FIVE PATIENTS)

Four patients had bilateral upper and lower lid ectropion as a result of severe lid skin shortage (Fig. 1), and two developed spontaneous eversion of the lids during sleep and on crying (Fig. 2). All had poor lid closure, with an inadequate blink response and signs of bilateral corneal exposure in the interpalpebral zone. Three patients, all of whom had had previous bilateral tarsorrhaphies which had broken down and failed to provide adequate corneal protection, developed permanent corneal scarring in the interpalpebral zone (Fig. 3); one of these had developed a corneal abscess. The ectropion was treated in all cases with full thickness skin grafting to correct the skin shortage, postauricular skin being used as the donor site (Fig. 4). In three patients all four lids were grafted and in the other patient postauricular Wolf grafts to the upper lids were combined with recession of the levator palpebrae superioris. Although there was skin shortage of the lower lids, it was not considered severe enough to warrant surgery. Four years later this patient required further surgery as result of corneal exposure. Consequently bilateral lower lid scleral grafts and skin grafts from the upper arm, together with bilateral

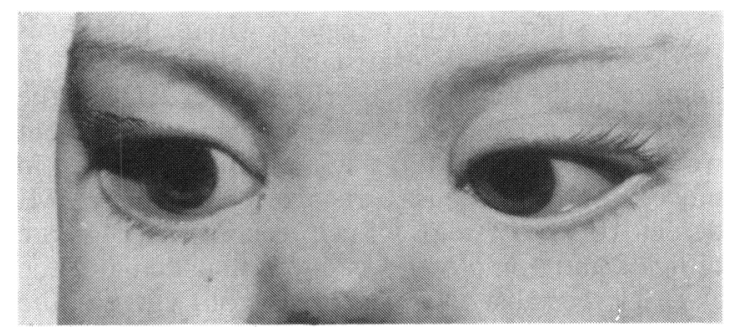

Fig. 1 Child with upper and lower lid ectropion. 


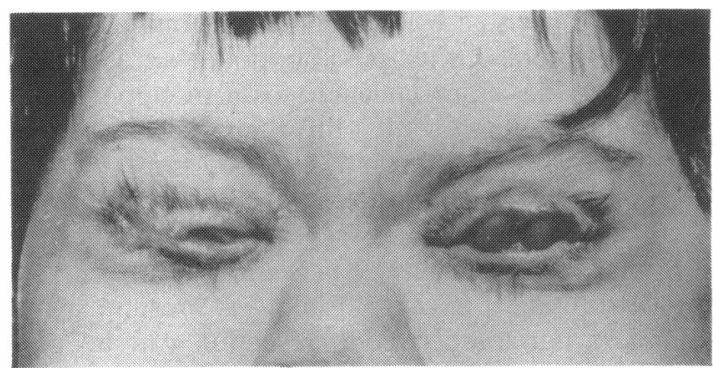

Fig. 2 Spontaneous eversion of the lids on crying.

lower lid shortening for excess lid laxity, were performed. In another patient lid skin grafting was combined with division of tarsorrhaphies and horizontal shortening of the lower lids (Fig. 5).

The fifth patient with ectropion in this group was a 55-year-old man. He had bilateral lower lid ectropion, bilateral blepharitis, madarosis, and corneal scarring in the interpalpebral area. However, the lid abnormalities were not considered severe enough to warrant surgery, and he was treated with ocular lubricants alone.

\section{ENTROPION GROUP (ONE PATIENT)}

An 11-year-old girl presented with epiphora. On examination she had bilateral upper and lower lid entropion more marked medially, with punctate staining of both corneas. The entropion was corrected surgically with an anterior lamellar repositioning of the upper lids and plication of the lower lid retractors (Jones type procedure).

TRICHIASIS GROUP (FOUR PATIENTS)

Four adult patients had trichiasis leading to surface corneal changes, but not associated with any malposition of the lid. Their ages were 15, 22, 27, and 54 years. In each case the trichiasis was associated with severe chronic blepharitis, and a variety of organisms were identified from lid margin cultures, including Staphylococcus epidermidis (three patients), Haemophilus influenzae (one patient), and Corynebacterium xerosis and a haemolytic strepto-

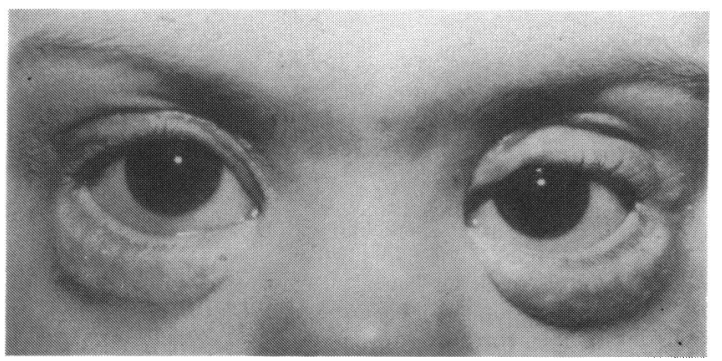

Fig. 4 Same patient as in Fig. 1 postoperatively.

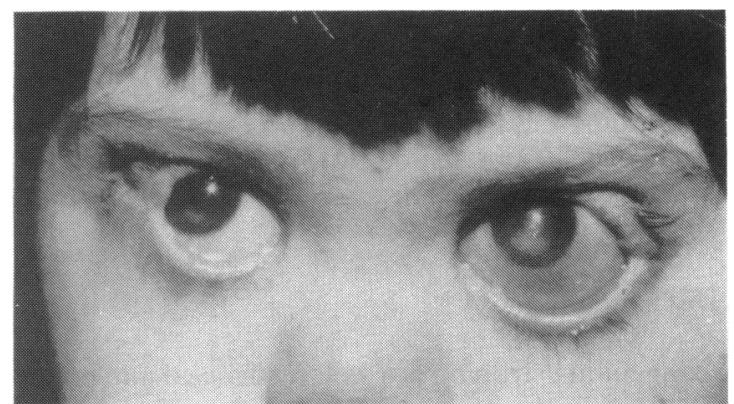

Fig. 3 Patient with upper and lower lid ectropion, bilateral tarsorrhaphies, and corneal scarring.

coccus (one patient). One of this group had been referred for corneal grafting for keratoconus but had been found to have trichiasis and lash ptosis which needed correction prior to corneal surgery. The trichiasis in these patients was treated with double freeze thaw cryotherapy to the lid margin. The lid was frozen from the lid surface with a special probe to a temperature of $-20^{\circ} \mathrm{C}$; the tissues were then allowed to thaw spontaneously and the freeze thaw cycle was repeated. The temperature was monitered with a thermocouple needle placed deep to orbicularis oculi and superficial to the tarsal plate in the region of the lash follicles. One patient had electrolysis to two isolated lashes on the right upper lid in addition to cryotherapy of the other lids.

\section{Results}

Ectropion group. In the four patients who underwent full thickness skin grafting the surgery corrected the ectropion as well as enabling good lid closure and improved corneal protection (Fig. 4). In all these cases the signs of corneal exposure resolved, though in three patients there was residual corneal scarring (Fig. 5). The cosmetic results of skin grafting in these young children were not good but improved with time. One patient in this group with ectropion, a 55year-old man, did not require surgery but responded well to treatment with ocular lubricants alone.

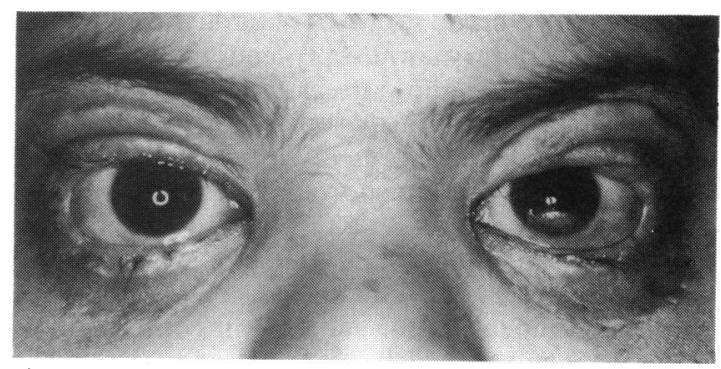

Fig. 5 Same patient as in Figs. 2 and 3 postoperatively. 
Entropion group. Surgery corrected both upper and lower lid entropion in this case.

Trichiasis group. Cryotherapy was effective in controlling the trichiasis in all four patients with no recurrences. The patient with keratoconus subsequently underwent successful bilateral penetrating keratoplasty.

\section{Discussion}

Congenital ectropion is a well recognised and potentially sight threatening condition seen in children with Down's syndrome but rarely other children. Many cases respond to conservative treatment alone, ${ }^{7}$ but in children with Down's syndrome surgical correction is often necessary. ${ }^{89}$ In this study four of our fifty-four patients had congenital ectropion leading to corneal exposure and requiring surgery. Two cases were severe enough to lead to spontaneous eversion of the lids, which increased on forced lid closure. In three patients lagophthalmos and associated corneal exposure led to corneal ulceration and permanent scarring; in one case it was severe enough to lead to a corneal abscess and hypopyon. It is therefore imperative that cases with significant corneal exposure are treated promptly with appropriate surgical techniques to prevent any permanent corneal complications.

In these patients the mechanism for the ectropion was skin shortage of the lids leading to vertical shortening of the anterior lid lamella, which pulls the lid margin away from the globe and results in ectropion. Associated conjunctival inflammation and chemosis further aggravate the condition, and in long-standing cases the problem may be compounded by the development of horizontal lid laxity.

Lateral tarsorrhaphies and horizontal lid shortening procedures have been used in an attempt to improve corneal cover but with variable results. ${ }^{89}$ Three of our patients had had previous tarsorrhaphies which had broken down, resulting in poor corneal protection, with one patient developing a corneal abscess and hypopyon as a result of corneal exposure. This technique should not be used in young children in whom the primary abnormality is skin shortage but reserved for cases of paralytic ectropion requiring temporary corneal protection.

We believe that the correct management of the congenital ectropion in these cases is correction of the underlying skin shortage with skin grafting. In most cases there is skin shortage of both upper and lower lids, and therefore transposition flaps cannot be used. Full thickness skin grafts are preferred and should be extended beyond the horizontal limb of the canthi to compensate for subsequent contraction of the graft. The preferred donor site is postauricular skin, and even in young babies adequate skin is usually present. Other possible donor sites include skin from the supraclavicular fossa or upper inner arm. Our results show that this is an effective way of correcting the ectropion and poor lid closure, thereby preventing corneal exposure.

In long-standing and severe cases additional surgical procedures may be necessary fully to correct the ectropion. If excess lid laxity is present, a full thickness horizontal wedge resection can be performed at the same time as skin grafting, or may be necessary if the ectropion recurs. In other cases skin grafting with or without a horizontal lid resection may be combined with a lid lengthening procedure if correction of the ectropion alone does not result in adequate lid cover. The upper lid may be lowered, provided the skin shortage is also corrected, by tarsotomy, recession of levator palpebrae superioris, or scleral grafting, depending on the degree of lid lengthening required. The lower lid may be raised by interposing a scleral graft between the anterior lid retractors and the lower border of the tarsus.

The patient with ectropion who did not require surgery was an adult with involutional ectropion, which was not therefore associated with skin shortage.

In the older patient with Down's syndrome chronic blepharoconjunctivitis may lead to the development of cicatricial entropion and trichiasis. Four of our patients were adults with long-standing blepharitis, and all showed signs of corneal disturbance as a result of the trichiasis. If neglected, such corneal changes may progress and corneal suppuration develop.

Electrolysis may be used for single or aberrant lashes, but for large numbers it is time consuming, poorly effective, and may produce scarring of the tarsal plate. These cases are better treated with double freeze thaw cryotherapy to the lid margin. ${ }^{10}$ More severe cases of trichiasis and those with cicatricial entropion may be treated surgically as appropriate for the degree of scarring. ${ }^{11}{ }^{12}$

This study highlights the specific malpositions seen in patients with Down's syndrome and outlines an approach to the management of these problems.

\section{References}

1 Patterson D. The causes of Down syndrome. Scientific American. 1987; 257 : 42-8.

2 Hohler $\mathrm{H}$. Changes in the facial expression as a result of plastic surgery in mongoloid children. Aesthetic Plast Surg 1977; 1: 24550.

3 Hatt M. Eyelid surgery in a child with Down's syndrome. Orbit 1987; 6: 65-7.

4 Lemperle G, Radu D. Facial plastic surgery in children with Down's syndrome. Plast Reconstr Surg 1980; 66: 337-44.

5 Rozner L. Facial plastic surgery for Down's syndrome. Lancet 1983; i: 1320-3. 
6 Olbrisch RR. Plastic surgical management of children with Down's syndrome: indications and results. Br J Plast Surg 1982; 35: 195-200.

7 Stillerman ML, Emanuel B, Padorr MP. Eversion of the eyelids in the newborn without an apparent cause. J Pediatr 1966; 69: 656-8.

8 Gilbert HD, Smith RE, Barlow MH, Mohr D. Congenital upper lid eversion and Down's syndrome. Am J Ophthalmol 1973; 75: 469-72.

9 Johnson CC, McGowan BL. Persistent congenital ectropion of all four eyelids with megaloblepharon. Am J Ophthalmol 1969; 67: $252-6$.

10 Johnson RLC, Collin JRO. Treatment of trichiasis with a lid cryoprobe. Br J Ophthalmol 1985; 65: 267-70.

$11 \mathrm{Kemp}$ EG, Collin JRO. Surgical management of upper lid entropion. Br J Ophthalmol 1986; 70: 575-9.

12 Collin JRO. A manual of systematic eyelid surgery. Edinburgh: Churchill Livingstone, 1983: 12-27.

Accepted for publication 3 November 1988. 